\title{
Genital Dysplasia and Immunosuppression: Why Organ-Specific Therapy Is Important
}

\author{
Raphael Sager $^{\mathrm{a}}$ Pascal Frei $^{\mathrm{b}}$ Urs C. Steiner ${ }^{\mathrm{c}}$ Daniel Fink $^{\mathrm{a}}$ Cornelia Betschart $^{\mathrm{a}}$ \\ ${ }^{a}$ Department of Gynecology, University Hospital of Zurich, Zurich, Switzerland; ${ }^{b}$ Gastroenterology, Clinic Bethanien, \\ Zurich, Switzerland; ' Department of Immunology, University Hospital of Zurich, Zurich, Switzerland
}

\section{Keywords}

Human papillomavirus - Genital dysplasia · Crohn's disease · TNF-a blockers

\begin{abstract}
Background: Young patients with Crohn's disease (CD) show a high prevalence of human papillomavirus (HPV) which is the main cause of high-grade squamous intraepithelial lesions (HSIL). A major complication for patients undergoing immunocompromising therapy is the development of genital dysplasia. Methods: We report the case of a 32-year-old patient with recurrent genital dysplasia under long-term therapy for CD with a focus on different drug-related, immunosuppressive mechanisms. Results: Gynecological examination and biopsy revealed high-grade vulvar intraepithelial neoplasia (VIN) positive for HPV 16 treated with laser vaporization. Due to the combination of HPV positivity, intraoperative multilocularity, and CD, follow-up examinations were performed every 6 months. One year later, the patient showed a VIN at a new location and additionally, a cervical intraepithelial neoplasia (CIN), which were surgically treated. Catch-up HPV vaccination was applied accessorily. After the switch from a TNF-a blocker to vedolizumab, which acts as a gut-selective anti-integrin, the subsequent PAP smear, vulvoscopy, and colposcopy showed no more evidence of dysplasia. Conclusions: This case report high-
\end{abstract}

lights that gut-selective immunosuppression with vedolizumab might be favorable in young HPV-positive patients due to a good side effect profile. Regular screening and HPV vaccination are a mainstay of dysplasia prevention and control. The risk for HPV-associated dysplasia in immunosuppressed patients is highly dependent on the choice of immunosuppressive therapy.

(c) 2019 S. Karger AG, Base

\section{Introduction}

Around $80 \%$ of sexually active adults will acquire a human papillomavirus (HPV) infection at least once in their lifetime. The highest prevalence of HPV infection in the female genital tract (up to $30 \%$ ) is found in 15 to 25 -yearold females due to a low immunization rate and the frequent switching of sexual partners relative to older age groups [1-3]. HPV is associated with several genital malignant cancer precursors including cervical intraepithelial neoplasia (CIN), vulvar intraepithelial neoplasia (VIN), vaginal intraepithelial neoplasia (VAIN), and anal intraepithelial neoplasia (AIN). These precancerous lesions are differentiated into low- and high-grade squamous intraepithelial lesions (LSIL, HSIL) [4-6]. The host immune system usually clears an HPV infection successfully.

\section{KARGER}

(C) 2019 S. Karger AG, Basel

E-Mail karger@karger.com

www.karger.com/iid
PD Dr. med. Cornelia Betschart

Department of Gynecology, University Hospital Zurich

Frauenklinikstrasse 10

CH-8091 Zurich (Switzerland)

E-Mail cornelia.betschart@usz.ch 


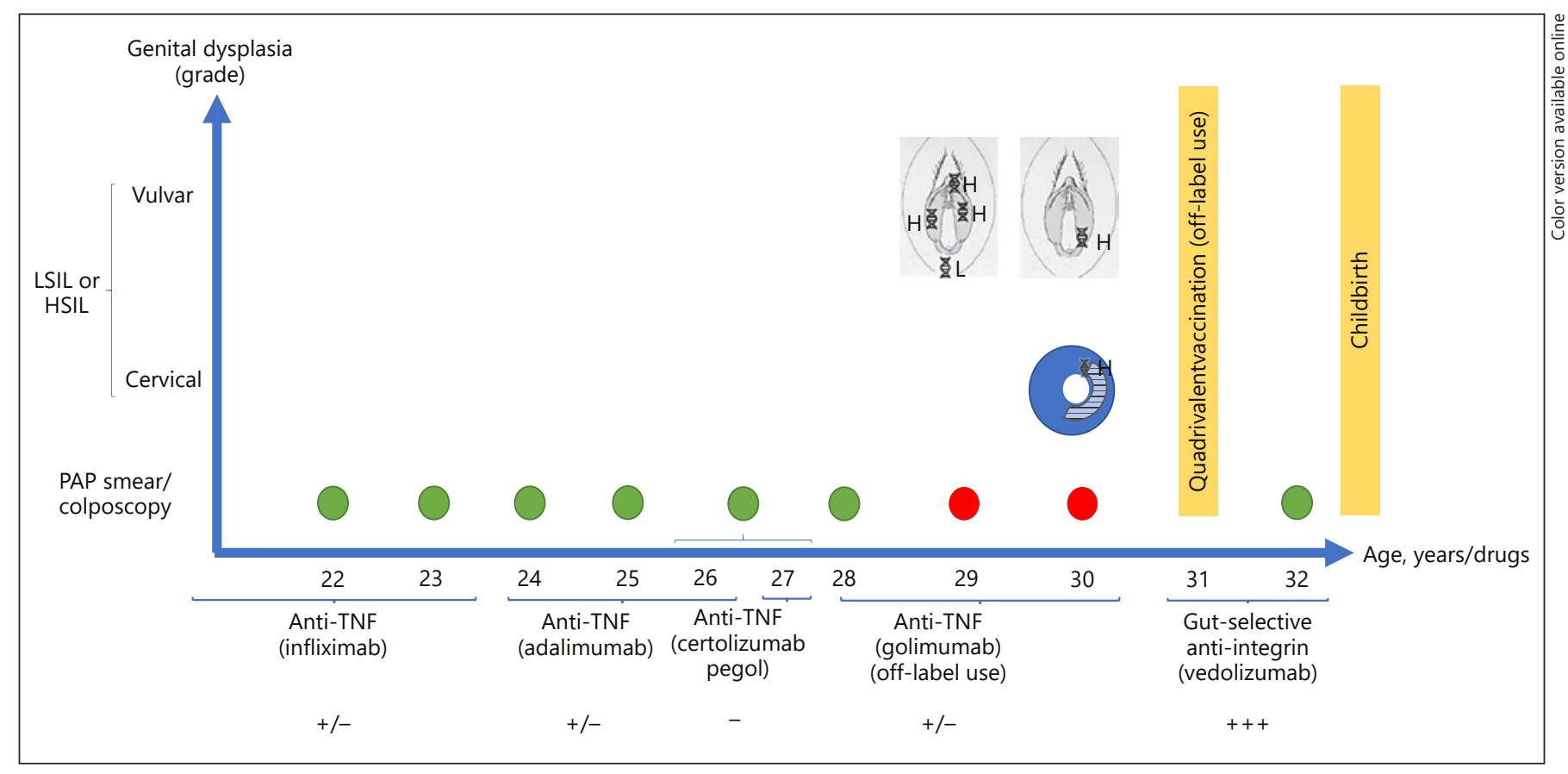

Fig. 1. Occurrence of HPV-associated dysplasia depending on the drug intake. The $x$ axis shows the timeline in years of age and chronological use of immunosuppressants, as well as the drug effect in a semiquantitative way from - (no effect) to +++ (maximum effect). The $y$ axis demonstrates vulvar and cervical dysplasia. Outlines of the vulva and cervix are shown, with sites of biopsy and dysplasia. H, high-grade lesion; L, low-grade lesion.

Patients with immunosuppressive drugs have an increased risk for persistent infections and progression to intraepithelial neoplasia [7]. The neoplasia risk differs depending on both the underlying disease and the mechanism of action of the immunosuppressant. In Crohn's disease (CD), which is one of the two main forms of inflammatory bowel disease, currently used immunosuppressive therapies include glucocorticosteroids, methotrexate, thiopurines, and biologicals. The most frequently used biologicals are tumor necrosis factor (TNF)- $\alpha$ blockers, ustekinumab and vedolizumab. Vedolizumab is a fully humanized monoclonal antibody that specifically blocks the migration of a subset of leukocytes harboring the $\alpha \beta 47$ integrin into inflamed intestinal tissue via MAdCAM-1. Those biologicals which work against cytokines or cell surface antigens are very potent therapeutics against autoimmune diseases, inflammatory diseases, and tumors. However, these biologicals can also induce adverse reactions including autoimmunity as well as bacterial, viral, or fungal infections $[8,9]$. Patients with HPV infection and inflammatory bowel disease have an increased prevalence for genital dysplasia [10-14].

Genital Dysplasia: Importance of

Organ-Specific Therapy
We aim to raise awareness of immunosuppression-associated and HPV-induced genital dysplasia and discuss possible pathomechanisms.

\section{Materials and Methods}

We describe the case of a 32-year-old female patient who was diagnosed with $\mathrm{CD}$ at the age of 22 years. We consider the patient's history, the course of the disease with a focus on the occurrence of genital dysplasia, and the clinical findings in colposcopy and histology. Different conservative and surgical treatments are discussed, with a focus on the role of the immunosuppressive side effects of the currently used biologicals. Recommendations for the follow-up care and disease management for young, female patients with $\mathrm{CD}$ are derived from both the presented clinical findings and from a review of the literature.

\section{Results}

The 32-year-old patient had a 10-year history of CD and presented with unilateral vulvar edema 8 years after the first CD manifestation. Initial CD manifestation was 

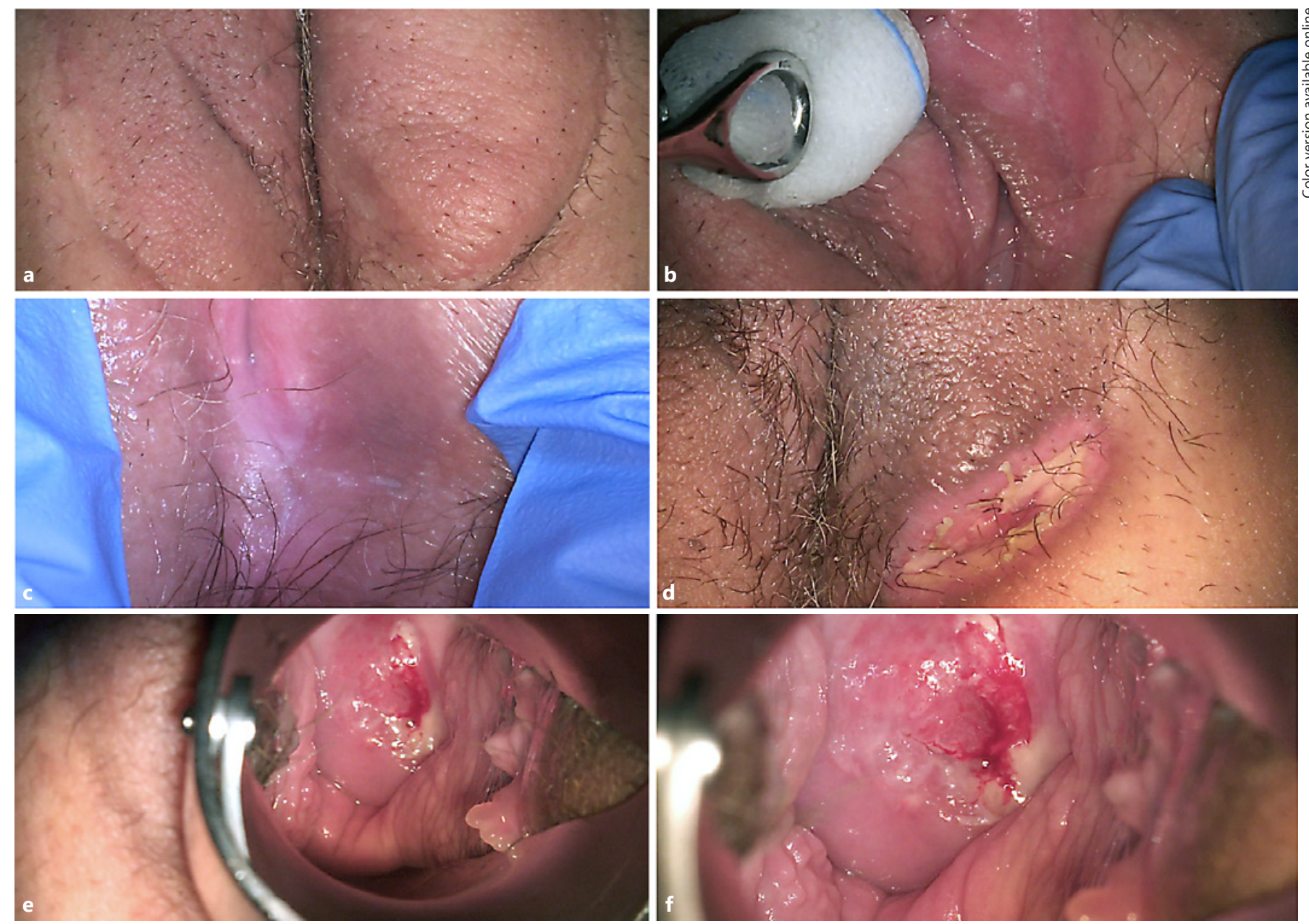

Fig. 2. Vulvoscopic and colposcopic presentation of different types of genital dysplasia in our patient. a Edema of the left labium majus. b Aceto-white leukoplakia of the left labium minus, histologically VIN III. c Aceto-white (5\%) leukoplakia of the perineum, histologically VIN III. d Lesion after excision and laser evaporization. e Colposcopy of the cervical lesion; biopsy revealed an HSIL at 2 o'clock. $f$ Cervical lesion (close-up); aceto-white patterns from 2 to 9 o'clock, corresponding to HSIL in the conization sample (R0).

a terminal ileitis, left-sided colitis, and perianal activity. For the first 5 years following the diagnosis, the patient received several immunosuppressive agents including glucocorticosteroids, thiopurines (short-term for 3-4 weeks in the first and fifth year of the disease; not tolerated due to adverse events such as headache, nausea, and arthralgia), and TNF- $\alpha$ blockers including 2 years of infliximab treatment, 2 years of adalimumab treatment, and 3 months of certolizumab pegol treatment (Fig. 1). Due to a steroid-refractory ileus, a laparoscopic ileocecal resection was performed at age 27 with postoperative complications. The recovery after the laparoscopic ileocecal resection was complicated due to anastomotic leakage of the ileo-transverse anastomosis, a fistula of the small bowel $50 \mathrm{~cm}$ after the angle of Treitz, a stomal stenosis, and an abscess in the right lower abdomen requiring two subsequent laparotomies. Due to an early CD relapse, golimumab was given as a fourth TNF-a blocker for 2 years (which it must be stated is an off-label treatment since golimumab is licensed for ulcerative colitis only). After 8 years of $\mathrm{CD}$ and after years of immunosuppression including thiopurines and four different TNF- $\alpha$ blockers, the patient developed a swelling of the left labium majus. An MRI showed no evidence of underlying fistulizing CD or granulomatous subcutaneous disease. The patient was bothered by the swelling which led to her avoiding sexual 
intercourse in a stable relationship. The patient was admitted to the gynecological outpatient clinic to rule out an immunosuppression-associated complication (infection or cancer). There was no evidence of an infectious disease. The size of the edema was demarcated by the left labium majus, and measured approximately $7.8 \times 3.2 \times$ $0.4 \mathrm{~cm}$ (Fig. 2a). A vulvo-colposcopy was performed with the application of aqueous 5\% acetic acid (Fig. 2b). A punch biopsy of the left labium minus was taken from an aceto-positive discrete keratosis that histologically showed a high-grade lesion (VIN III), which was HPV 16 positive (Fig. 2b). In this examination, the VIN was detected as an incidental finding but as a potentially malignant disease. No biopsy was undertaken from the soft, smooth, and even edema, as no genital granulomatosis was clinically suspected in the pelvic MRI. Elephantiasis with filarial parasites did not align with the personal history. As the edema was self-limiting and did not recur, no further edema-specific examinations were considered necessary.

A lymphedema of the left labium majus was postulated due to several abdominal surgeries (Fig. 2a). However, the last abdominal surgery (a colonic resection of the transverse colon and a reversal of an ileal stoma) had been performed 3 years previously.

In the subsequent surgery, VIN III was intraoperatively excised on both sides of the labium minus, and a biopsy in the introitus revealed a further low-grade intrasquamous lesion (VIN I, LSIL). Histological examination showed dysplasia-free margins (R0). Laser vaporization of the surroundings of the vulvar lesions and introital biopsy were performed. The immunosuppression with golimumab as a fourth TNF- $\alpha$ blocker was continued due to the lack of alternatives at that time. The follow-up examination after 6 months was unremarkable. Another 6 months later, the follow-up examination showed a high-grade VIN (VIN II-III) lesion at the left labium majus, in the posterior part, without edema (Fig. 2c). Therefore, a fusiform excision of the left labium major, a perineal biopsy, laser vaporization of the vulva, a punch biopsy of the cervix, and an anoscopy were carried out (Fig. 2d).

Histology of the cervix also revealed an HSIL (Fig. 2e, f), that was removed by the loop electrosurgical excision (LEEP) procedure. Additionally, an HPV catch-up vaccination with the quadrivalent agent Gardasil was administered [15]. At that time, the immunosuppressive agent was switched to vedolizumab as a gut-selective immunosuppressive agent, which had newly become available in Switzerland.

Genital Dysplasia: Importance of

Organ-Specific Therapy
Follow-up examinations carried out over the following 21 months, and while using the vedolizumab-based treatment, showed no further abnormalities in either the PAP smears or in the aceto-white testing. At age 32, and 1.5 years after the first VIN treatment, the patient became pregnant. After two miscarriages, both in the first trimester, the third pregnancy took an obstetrically normal course. Colposcopic and cytological examinations did not reveal any dysplasia. Continued treatment with vedolizumab and mesalazine before pregnancy and in the first trimester of pregnancy could not prevent a small intestinal ileus due to fibrostenotic CD. A second small intestinal resection $(17.5 \mathrm{~cm})$ with postoperative anastomotic leakage and ileostomy was performed in the second trimester. Despite all the medication and surgical complications, the patient delivered a healthy baby in the 37 th gestational week $(2,770 \mathrm{~g}$, $46 \mathrm{~cm})$.

\section{Discussion}

$\mathrm{CD}$ is a chronic inflammatory bowel disease affecting young patients [16]. CD often coincides with the fertile phase which is also the most vulnerable phase for HPV infections [3]. This case study supports previous studies that highlighted the role of immunosuppressive agents in the development of multilocular genital dysplasia in CD patients [17-19], while providing new insight into the beneficial role of a gut-selective immunosuppression therapy.

CD itself may be associated with a greater risk of genital dysplasia, especially for VIN $[12,18]$. Immunosuppressive treatments, which are necessary in most patients, may additionally have an impact on infection risk and HPV progression. Azathioprine increases the risk of genital dysplasia in a dose-dependent manner [20]. In our patient the high-grade lesions occurred under longstanding therapy with different TNF- $\alpha$ blockers (Fig. 2). Genital dysplasia has been described after the use of TNF- $\alpha$ blockers $[21,22]$. TNF- $\alpha$ plays an important role in the immune response to infections. After application of anti-TNF- $\alpha$ monoclonal antibodies, activated T lymphocytes and macrophages fall into apoptosis in vivo [2325]. Literature concerning HPV infections under TNF- $\alpha$ blockers is scarce. However, it has been shown that HPVspecific CD4+ and CD8+ T cells are important for longterm tumor protection [26]. We assume that the immunosuppression played an important role in the rapid progression of the condition to genital high-grade dysplasia

Inflamm Intest Dis 2019;4:154-160 157 
at a young age and to the de novo occurrence of highgrade lesions at different sites.

The most interesting finding of our case, however, is that the HPV-associated precancerous lesions disappeared in a follow-up performed 2 years after the switch from unselective immunosuppression with TNF- $\alpha$ blockers to the gut-selective antibody vedolizumab. Careful follow-up controls with PAP smears and vulvo-colposcopy including aceto-white testing were normal. Vedolizumab shows a strong or intense binding capacity to the small intestine and colon, respectively, as the main targets for CD treatment $[27,28]$, but no or only a weak binding capacity to the skin (vulva) and the cervix, respectively [20]. Our findings suggest that, in the context of genital dysplasia, it is favorable to use either inflammatory bowel disease treatments that are less immunosuppressive (avoiding TNF- $\alpha$ blockers) or specific immunosuppressive treatments (including the gut-selective antibody vedolizumab).

Other potentially relevant risk factors for a more rapid disease progression are age over 25 years, HSIL of the cervix, and a history of an HPV type 16 infection [29]. The occurrence and recurrence of genital dysplasia in our case were certainly influenced by these different risk factors.

Routine PAP smears are performed every 3 years in the base Swiss population. In the context of female CD patients, the ECCO guidelines strongly recommend regular gynecologic screening for cervical cancer, especially if the patient is also being treated with immunosuppressive/ immunomodulating drugs. Our case highlights that gynecologic screening in CD patients should include careful vulvoscopy to exclude VIN as well. We propose an annual screening for CD patients (PAP smear plus HPV PCR) as well as a vulvo-colposcopy of the anogenital region with aceto-white testing for the early detection of anogenital lesions. In the case of posttreatment, we propose follow-up visits every 6 months.

The initial vulvar edema was self-limiting in our patient. In the literature the underlying pathomechanism of vulvar edema is described as a chronic granulomatous inflammation of the skin which is one of the extra-intestinal manifestations of CD [30]. However, histologies in our patient showed no evidence of dermal epithelioid granulomas.

Based on the gynecological findings and locations, as seen in Figure 1, the treatment had to be individually adjusted since laser vaporization, excision, and conization have different risk profiles. To find the suitable treatment choice, the staging of the lesion, the age of the patient, and the cytology should all be considered [31].
For instance, cervical conization needs to be highlighted in the context of this case, since our patient had a desire to become pregnant. This is due to the fact that the height of the conus has a major impact on the risk of preterm delivery. A significant increase for preterm delivery was found at a conus height of $1.5 \mathrm{~cm}$. The LEEP procedure showed the best outcome (lowest risk) regarding a preterm delivery and is therefore the gold standard [32,33]. Standard practice in preconception counseling after a conization with dysplasia-free margins (R0) is to defer pregnancy for 6 months in order to prove a negative PAP smear.

The additional quadrivalent HPV vaccination after the conization was performed to improve the outcome after the different surgical treatments and also to improve the patient's immunity. The determinant factor for this approach is a proven lower relapse rate of about 35.2\% [34]. Vaccination might have contributed to the favorable follow-up. There are three currently available vaccines: divalent targeting HPV 16/18, quadrivalent targeting HPV $6 / 11 / 16 / 18$, and nonvalent targeting the aforementioned types as well as HPV types 31/33/45/51/58. The vaccines demonstrated a potential to prevent $70 \%$ of cervical cancer within an adequately vaccinated population. In the future, it is expected that the nonvalent vaccines achieve an $85-95 \%$ protection against VIN and a $90 \%$ protection against CIN $[35,36]$. The American Society for Colposcopy and Cervical Pathology (ASSCP) recommends that the most effective primary prevention is to vaccinate girls before they become sexually active [37].

A better understanding of the pathways of HPV infection and immunity is crucial in order to be able to develop individualized recommendations. Given the fact that there is still a knowledge gap, HPV vaccination of immunocompromised patients and patients with an iatrogenic immunocompromising therapy is crucial. Various immunosuppressive agents including biologicals have to be followed meticulously regarding their effects on genital dysplasia in order to prevent cervical, vulvar, vaginal, or anal cancer.

\section{Conclusion}

Our case study demonstrated the recurrence of HPVassociated multilocular genital dysplasia under TNF- $\alpha$ blockers. The genital dysplasia was detected due to a good collaboration between different medical specialties. The early detection in combination with an individualized therapy including surgery, vaccination, and adjustment of immunosuppression therapy led to a favorable outcome. 


\section{Statement of Ethics}

Written informed consent was obtained from the patient for the publication of any data and accompanying images.

\section{Disclosure Statement}

The authors declare that they have no competing interests. Pascal Frei has received consulting fees from MSD, AbbVie, and Takeda and travel grants from Vifor Pharma. Takeda, which is marketing vedolizumab, was not involved in this case report and had thus no influence on the paper.

\section{References}

1 Koutsky L. Epidemiology of genital human papillomavirus infection. Am J Med. 1997 May; 102 5A:3-8.

2 Bosch FX, Burchell AN, Schiffman M, Giuliano AR, de Sanjose S, Bruni L, et al. Epidemiology and natural history of human papillomavirus infections and type-specific implications in cervical neoplasia. Vaccine. 2008 Aug;26 Suppl 10:K1-16.

3 Remschmidt C, Fesenfeld M, Kaufmann AM, Deleré Y. Sexual behavior and factors associated with young age at first intercourse and HPV vaccine uptake among young women in Germany: implications for HPV vaccination policies. BMC Public Health. 2014 Dec;14: 1248.

4 Lillo FB. Human papillomavirus infection and its role in the genesis of dysplastic and neoplastic lesions of the squamous epithelia. New Microbiol. 2005 Apr;28(2):111-8.

5 Burd EM, Dean CL. Human Papillomavirus. Microbiol Spectr. 2016 Aug;4(4):4.

6 Darragh TM, Colgan TJ, Thomas Cox J, Heller DS, Henry MR, Luff RD, et al.; Members of the LAST Project Work Groups. The Lower Anogenital Squamous Terminology Standardization project for HPV-associated lesions: background and consensus recommendations from the College of American Pathologists and the American Society for Colposcopy and Cervical Pathology. Int J Gynecol Pathol. 2013 Jan;32(1):76-115.

7 Satmary W, Holschneider CH, Brunette LL, Natarajan S. Vulvar intraepithelial neoplasia: risk factors for recurrence. Gynecol Oncol. 2018 Jan;148(1):126-31.

8 Davis BP, Ballas ZK. Biologic response modifiers: Indications, implications, and insights. J Allergy Clin Immunol. 2017 May; 139(5): 1445-56.

9 Fernández-Ruiz M, Meije Y, Manuel O, Akan $\mathrm{H}$, Carratalà J, Aguado JM, et al. ESCMID Study Group for Infections in Compromised Hosts (ESGICH) Consensus Document on the safety of targeted and biological therapies: an infectious diseases perspective (Introduction). Clin Microbiol Infect. 2018 Jun;24 Suppl 2:S2-9.

10 Foo WC, Papalas JA, Robboy SJ, Selim MA. Vulvar manifestations of Crohn's disease. Am J Dermatopathol. 2011 Aug;33(6):588-93.
11 Vuitton L, Jacquin E, Parmentier AL, Crochet E, Fein F, Dupont-Gossart AC, et al. High Prevalence of Anal Canal High-Risk Human Papillomavirus Infection in Patients with Crohn's Disease. Clin Gastroenterol Hepatol. 2018 Nov; 16(11):1768-1776.e5.

12 Li M, Han Y, Wu TT, Feng Y, Wang HB. Tumor necrosis factor alpha rs1800629 polymorphism and risk of cervical lesions: a metaanalysis. PLoS One. 2013 Aug;8(8):e69201.

13 Hill-Daniel J, Roett MA. Genital cancers in women: vulvar cancer. FP Essent. 2015 Nov; 438:31-43.

14 Dederichs F, Iesalnieks I, Sladek M, Tzivinikos C, Hansen R, Muñoz C, et al.; ECCOCONFER investigators. Genital Granulomatosis in Male and Female Patients With Crohn's Disease: Clinical Presentation and Treatment Outcomes. J Crohn's Colitis. 2018 Jan;12(2):197-203.

15 Castellsagué X, Ault KA, Bosch FX, Brown D, Cuzick J, Ferris DG, et al. Human papillomavirus detection in cervical neoplasia attributed to 12 high-risk human papillomavirus genotypes by region. Papillomavirus Res. 2016 Dec;2:61-9.

16 Burisch J, Munkholm P. The epidemiology of inflammatory bowel disease. Scand J Gastroenterol. 2015 Aug;50(8):942-51.

17 Thomson AB, Gupta M, Freeman HJ. Use of the tumor necrosis factor-blockers for Crohn's disease. World J Gastroenterol. 2012 Sep;18(35):4823-54

18 Annese V, Beaugerie L, Egan L, Biancone L, Bolling C, Brandts C, et al.; ECCO. European evidence-based consensus: inflammatory bowel disease and malignancies. J Crohn's Colitis. 2015 Nov;9(11):945-65.

19 Matsuo K, Chi DS, Eno ML, Im DD, Rosenshein NB. Vulvar mucinous adenocarcinoma associated with Crohn's disease: report of two cases. Gynecol Obstet Invest. 2009;68(4):2768.

20 Dugué PA, Rebolj M, Hallas J, Garred P, Lynge E. Risk of cervical cancer in women with autoimmune diseases, in relation with their use of immunosuppressants and screening: population-based cohort study. Int J Cancer. 2015 Mar;136(6):E711-9.

21 Antoniou C, Kosmadaki MG, Stratigos AJ, Katsambas AD. Genital HPV lesions and molluscum contagiosum occurring in patients receiving anti-TNF- $\alpha$ therapy. Dermatology. 2008;216(4):364-5.
22 Bhatia J, Bratcher J, Korelitz B, Vakher K, Mannor S, Shevchuk M, et al. Abnormalities of uterine cervix in women with inflammatory bowel disease. World J Gastroenterol. 2006 Oct;12(38):6167-71.

23 Bruns H, Meinken C, Schauenberg P, Härter G, Kern P, Modlin RL, et al. Anti-TNF immunotherapy reduces $\mathrm{CD} 8+\mathrm{T}$ cell-mediated antimicrobial activity against Mycobacterium tuberculosis in humans. J Clin Invest. 2009 May;119(5):1167-77.

24 Atreya R, Zimmer M, Bartsch B, Waldner MJ, Atreya I, Neumann $\mathrm{H}$, et al. Antibodies against tumor necrosis factor (TNF) induce T-cell apoptosis in patients with inflammatory bowel diseases via TNF receptor 2 and intestinal CD14凶 macrophages. Gastroenterology. 2011 Dec;141(6):2026-38.

25 Mitoma H, Horiuchi T, Tsukamoto H, Ueda N. Molecular mechanisms of action of antiTNF- $\alpha$ agents - Comparison among therapeutic TNF- $\alpha$ antagonists. Cytokine. 2018 Jan;101:56-63.

26 Furniss CS, McClean MD, Smith JF, Bryan J, Nelson HH, Peters ES, et al. Human papillomavirus 16 and head and neck squamous cell carcinoma. Int J Cancer. 2007 Jun;120(11): 2386-92.

27 Dubinsky MC, Cross RK, Sandborn WJ, Long M, Song X, Shi N, et al. Extraintestinal Manifestations in Vedolizumab and Anti-TNFTreated Patients with Inflammatory Bowel Disease. Inflamm Bowel Dis. 2018 Apr;24(9): 1876-82.

28 Tadbiri S, Peyrin-Biroulet L, Serrero M, Filippi J, Pariente B, Roblin X, et al.; GETAID OBSERV-IBD study group. Impact of vedolizumab therapy on extra-intestinal manifestations in patients with inflammatory bowel disease: a multicentre cohort study nested in the OBSERV-IBD cohort. Aliment Pharmacol Ther. 2018 Feb;47(4):485-93.

29 Cubo-Abert M, Centeno-Mediavilla C, Franco-Zabala P, Merced-Vázquez C, Castellví J, García A, et al. Risk factors for progression or persistence of squamous intraepithelial lesions diagnosed during pregnancy. J Low Genit Tract Dis. 2012 Jan;16(1):34-8.

30 Ghosh D, Woodrow S, Mathew J, Lopes A, Das N. Chronic granulomatous inflammation of the vulva: an unusual presentation with diagnostic and therapeutic difficulties. J Low Genit Tract Dis. 2011 Oct;15(4):322-4. 
31 Jeronimo J, Castle PE, Temin S, Denny L, Gupta V, Kim JJ, et al. Secondary Prevention of Cervical Cancer: ASCO Resource-Stratified Clinical Practice Guideline. J Glob Oncol. 2016 Oct;3(5):635-57.

32 Kristensen J, Langhoff-Roos J, Kristensen FB. Increased risk of preterm birth in women with cervical conization. Obstet Gynecol. 1993 Jun;81(6):1005-8.

33 Liu Y, Qiu HF, Tang Y, Chen J, Lv J. Pregnancy outcome after the treatment of loop electrosurgical excision procedure or coldknife conization for cervical intraepithelial neoplasia. Gynecol Obstet Invest. 2014;77(4): 240-4.

34 Joura EA, Garland SM, Paavonen J, Ferris DG, Perez G, Ault KA, et al.; FUTURE I and II Study Group. Effect of the human papillomavirus (HPV) quadrivalent vaccine in a subgroup of women with cervical and vulvar disease: retrospective pooled analysis of trial data. BMJ. 2012 Mar;344:e1401.

35 Pitisuttithum P, Velicer C, Luxembourg A. 9-Valent HPV vaccine for cancers, pre-cancers and genital warts related to HPV. Expert Rev Vaccines. 2015;14(11):1405-19.
36 Cuzick J. Gardasil 9 joins the fight against cervix cancer. Expert Rev Vaccines. 2015;14(8): 1047-9.

37 Saslow D, Solomon D, Lawson HW, Killackey M, Kulasingam SL, Cain J, et al.; American Cancer Society; American Society for Colposcopy and Cervical Pathology; American Society for Clinical Pathology. American Cancer Society, American Society for Colposcopy and Cervical Pathology, and American Society for Clinical Pathology screening guidelines for the prevention and early detection of cervical cancer. Am J Clin Pathol. 2012 Apr;137(4):516-42. 